\title{
Morphological and Mechanical Properties of Nylon 6 Gel and Melt Films
}

\author{
Masaru MATSUO* and Kyoko INABA \\ Department of Clothing Science, Faculty of Home Economics, \\ Nara Women's University, Nara 630, Japan
}

(Received December 3, 1987)

\begin{abstract}
Nylon 6 films were prepared by gelation/crystallization from solutions and by molding from melt state. The resultant gel and melt films were elongated in a hot oven at $205^{\circ} \mathrm{C}$ under nitrogen. As co-solvent mixtures in preparing gel films, the formic acid/chloroform compositions studied were $100 / 0,75 / 25$, and 50/50. The mechanical properties of the gel films were hardly affected by composition but the gel film with the $75 / 25$ composition could be readily elongated in comparison with those with the $100 / 0$ and 50/50. The Young's moduli and the tensile moduli for all the specimens are less than 5 and $0.15 \mathrm{GPa}$, respectively. The values of the Young's moduli are much lower than the crystal lattice modulus $(183 \mathrm{GPa})$ of nylon 6 . To determine the reason for the poor mechanical properties of these specimens, the morphological properties of the gel film with the 75/25 composition, as an example, were investigated in comparison with those of the melt film by using wide angle X-ray diffraction, small angle X-ray scattering, and small angle light scattering. Deformation was found to be independent of a crystal transformation from a folded to a fibrous type and the preferential orientation of the crystal fiber axis (the $b$-axis) is mainly due to the rotation of crystallites leading to taut tie molecules. This mechanism hampers ultradrawing of nylon 6 films to realize high modulus and high strength.
\end{abstract}

KEY WORDS Nylon 6/Gel Film / Melt Film / Formic Acid / Chloroform /

Since 1974, the preparation of polymeric fibers and films with high modulus and high strength has been extensively investigated on the basis of the surface growth and gel deformation method and results of interest have been obtained for polyethylene ${ }^{1-8}$ and polypropylene. $^{8,9}$ The surface growth method has been developed by Pennings et al. ${ }^{1}$ and Keller et $a .^{2}$ to produce high strength and high modulus polyethylene fibers. According to their reports, the best values of Young's modulus and tensile strength were $142 \mathrm{GPa}^{1}$ and $4.7 \mathrm{GPa}$, respectively. The deformations of single crystal mats ${ }^{3,4}$ and gels have been wellknown as an ultradrawing method. ${ }^{5-7}$ In most of cases, they were prepared by crystallization from dilute solutions using polyethylenes with molecular weights greater than $1 \times 10^{6}$. Recently, Matsuo et al. produced ultradrawn polyethylene $^{7}$ and polypropylene ${ }^{8}$ whose Young's moduli at $20^{\circ} \mathrm{C}$ were 216 and 40.4 $\mathrm{GPa}$, respectively, according to the method of Smith and Lemstra. ${ }^{4-6}$ These values were nearly equal to the crystal lattice moduli of polyethylene $^{7}$ and polypropylene ${ }^{9}$ measured by X-ray diffraction technique.

Unfortunately, the application range of poly- $\alpha$-olefine fibers is limited by the flexibility of the individual chains and low melting point. Therefore, it is important to achieve ultradrawing of other crystalline polymers such as polyesters and polyamides with melting points higher than $200^{\circ} \mathrm{C}$ and with high molecular rigidity. Nylon 6 fibers are widely used, e.g., as

\footnotetext{
* To whom correspondence should be addressed.
} 
tyre yarns and attempts have been made to increase the modulus. Nylon 6, however, is certainly among the most difficult to draw to high draw ratio and to realize high Young's modulus which is sufficiently enough for commercial thermoplastics. The difficulty of ultradrawing of nylon 6 is largely due to the presence of intermolecular hydrogen bonding between adjacent amide groups. They act as quasi-crosslinks, inhibiting the sliding of chains along the hydrogen bond plane during drawing. In order to remove this defect, dry and gel spinnings have been developed for production of nylon 6 .

Chuah and Porter ${ }^{10}$ attempted to prepare a gel by disolving nylon 6 in benzylalchohol at $165^{\circ} \mathrm{C}$ and cooling to room temperature. A resultant partially dried gel film was drawn by co-extrusion with poly(oxymethylene) as the outer billet at $150^{\circ} \mathrm{C}$ in an Instron rheometer up to a maximum draw ratio of 5.7. The drawn gel film showed double orientations of crystallites at initial drawing; One is the preferential orientation of the crystal chain axes being parallel to the drawn direction and the other is the orientation of chain axes being perpendicular to the drawn direction. The preferential orientation of the crystal axes with respect to the drawn direction was raised at a draw ratio of 4.6 at the expense of the latter orientation. The Young's moduli of the film with such a characteristic crystal orientation were about $5.7 \mathrm{GPa}$ at draw ratios above 3.7.

On the other hand, Gololewski and Pennings ${ }^{11}$ produced nylon 6 filaments by dryspinning of solutions of nylon 6 in cosolvent mixtures of formic acid and chloroform followed by hot-drawing at $200-240^{\circ} \mathrm{C}$. The Young's modulus and tensile strength of the resultant nylon 6 fibers were strongly affected by the draw ratio, molecular weight, and kinds of spinning solutions and the best values reached 19 and $1 \mathrm{GPa}$, respectively. These values are highest among the values reported already but the Young's modulus is much lower than the crystal lattice modulus (183
GPa) estimated by the X-ray diffraction by Kaji and Sakurada. ${ }^{12}$

In this paper, the deformation mechanism of nylon 6 is studied using two kinds of films. One is prepared by gelation/crystallization from solutions with cosolvent mixtures of formic acid and chloroform and the other is prepared by molding from melt state. The focus is concentrated on what is the main factor hampering ultradrawing of nylon 6 to realize high modulus and high strength. In order to solve this problem in terms of morphological aspects, the investigation was done using wide angle X-ray diffraction (WAXD), small angle $X$-ray scattering (SAXS), small angle light scattering (SALS) under $H_{\mathrm{v}}$ polarization condition, birefringence, and differential scanning calorimetry (DSC).

\section{EXPERIMENTAL}

Nylon 6 with weight average molecular weight of $6.3 \times 10^{4}$ was used as the specimen. Viscosities to obtain the molecular weight were measured in $m$-cresol at $25^{\circ} \mathrm{C}$ and the molecular weight was calculated according to the relationship by Markhorst and Goldhart, ${ }^{13}$ as reported by Gogolewski and Pennings. ${ }^{11}$

In order to make clear the relationship between the physical properties of resultant gel films and the viscosity of solutions to prepare the gels, viscosity measurements were carried out using three kinds of solvents as a medium of formic acid-chloroform. The formic acid/ chloroform compositions, the F/C compositions, chosen were $100 / 0,75 / 25$, and $50 / 50$ as co-solvent mixtures. The solutions were stabilized with $0.1 \%(\mathrm{w} / \mathrm{w})$ anti-oxidant, 3-methyl6-tert-butylphenol.

Gels were prepared by crystallization from dilute solutions. The homogenized solution at a suitable concentration was poured into a glass plate and the plate was dipped into a water bath at $25-30^{\circ} \mathrm{C}$ to promote coagulation. The fresh gel films thus obtained were leached in running water for two days and 
dried at ambient condition. All the specimens were vacuum-dried for three days prior to all measurements in order to remove water as possible.

Melt films were prepared from nylon 6 chips to compare the mechanical properties with those of gel films. The chips were sandwiched between Teflon sheets at $245^{\circ} \mathrm{C}$ for $15 \mathrm{~min}$ at a pressure of $0.2 \mathrm{GPa}$. The molten sample was cooled to room temperature quickly.

Gel and melt films were stretched in a hot oven at $205^{\circ} \mathrm{C}$ under nitrogen. Prior to elongation, the specimens were annealed for 30 $\min$.

Densities of the gel and melt films were measured by a pycnometer in carbon tetrachloride-toluene as a medium. Prior to measuring the density, the films were cut into fragments and immersed in an excess of ethanol for a week in an ultra-sonic washing instrument and subsequently vacuum-dried for three days.

The melting point was estimated in terms of melting endotherm of DSC curves. Dry gels, weighing in $5 \mathrm{mg}$ each, were placed in a standard aluminum sample pan. Samples were heated at a constant rate of $10^{\circ} \mathrm{C} \mathrm{min}{ }^{-1}$.

Light scattering patterns were obtained with a 3-mW $\mathrm{He}-\mathrm{Ne}$ gas laser as the light source. Diffuse surfaces were avoided by sandwiching the specimens between microcover glasses with a silicon immersion oil having a similar index.

The X-ray measurements were carried out with a $12 \mathrm{~kW}$ rotating-anode $\mathrm{X}$-ray generator (Rigaku RDA-rA operated at $200 \mathrm{~mA}$ and $40 \mathrm{kV}$ ). SAXS patterns were obtained by point focusing with a three-pin hole collimator system. The X-ray exposure time was $50 \mathrm{~h}$. WAXD patterns were obtained with a flat camera and the intensity distribution was measured at a desired step interval of twice the Bragg angles with a desired time interval. The

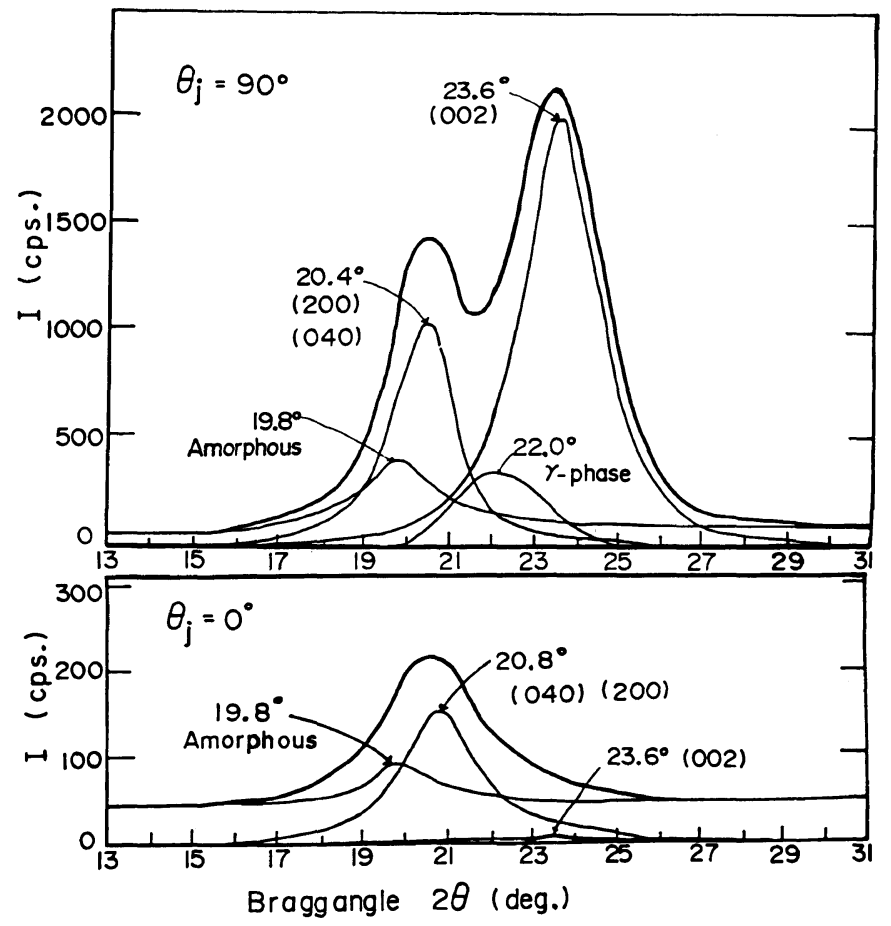

Figure 1. The contributions of the (200), (040), and (002) planes of the $\alpha$-form crystals, amorphous phase, and the $\alpha$-form crystals to the total X-ray diffraction curve at $\theta_{j}=90^{\circ}$ and $0^{\circ}$ for the gel film with $\lambda=4$. 
$\mathrm{X}$-ray beam was monochromatized with a curved graphite monochromater.

Orientation of crystallites was estimated in terms of the second order orientation factors from X-ray diffraction intensity distribution $I\left(2 \theta_{\mathbf{B}}, \theta_{j}\right)$ of the reciprocal lattice vector of the $j$-th crystal plane. The distribution function $I\left(\theta_{\mathrm{B}}, \theta_{j}\right)$ is given as a function of the angle $\theta_{j}$ denoting polar angle between the $j$-th reciprocal lattice vector and the stretching direction. The distribution is independent of the azimuthal angle, since the crystallites have random orientation with respect to the stretching direction. Therefore we have

$$
2 \pi q_{j}\left(\cos \theta_{j}\right)=\frac{\int_{2 \theta_{1}}^{2 \theta_{2}} I\left(2 \theta_{\mathbf{B}}, \theta_{j}\right) \mathrm{d} \theta_{\mathbf{B}}}{\int_{0}^{\pi}\left[\int_{2 \theta_{1}}^{2 \theta_{2}} I\left(2 \theta_{\mathbf{B}}, \theta_{j}\right) \mathrm{d} \theta_{\mathbf{B}}\right] \sin \theta_{j} \mathrm{~d} \theta_{j}}
$$

The function of $2 \pi q_{j}\left(\cos \theta_{j}\right)$ is determined from $I\left(2 \theta_{\mathrm{B}}, \theta_{j}\right)$ by the integral of $\mathrm{X}$-ray diffraction intensity distribution as a function of $2 \theta_{\mathbf{B}}$ for the equatorial direction as a fixed value of $\theta_{j}$. The change in $2 \pi q_{j}\left(\cos \theta_{j}\right)$ with $\theta_{j}$ may be obtained by the rotation of the specimen around its thickness direction. The actual measurements of the crystal orientation of nylon 6 were carried out by the (200), (002), and (040) planes of the $\alpha$-form crystals. The derivation of diffraction intensities from the (200), (002), and (040) planes from the total intensity was performed in the following manner. The total diffraction was first measured as a function of $2 \theta_{\mathbf{B}}$ over the range from 13 to $32^{\circ}$, after subtracting air-scattering and background noise. After then, the contributions of the (200) and (002) planes of the $\alpha$-form crystals, amorphous phases, and the superposed peak concerning (001) and (200) diffractions of the $\gamma$-form crystals ${ }^{14}$ (or the $\beta$-crystals ${ }^{15}$ ) were separated from the total $\mathrm{X}$-ray diffraction intensity. This treatment was accomplished by a method of trial and error. Figure 1 shows the examples of $\theta_{j}=90$ and $0^{\circ}$ for the gel film with the $75 / 25$ composition drawn to $\lambda=4$. As shown in the profile at $\theta_{j}=90^{\circ}$, each contribution was assumed to have a symmetrical distribution along $2 \theta_{\mathrm{B}}$ with the maximum peaks at 20.4 and $23.6^{\circ}$ for the (200) and (002) diffractions, respectively, of the $\alpha$-form crystals and with a maximum peak at $22.0^{\circ}$ for the superposition of the (001) and (200) diffraction peaks of the $\gamma$-form crystals. The maximum peak positions at 20.4 and $23.6^{\circ}$ for the (200) and (002) diffractions are different from the theoretical values, 20.2 and $24.0^{\circ}$, respectively, calculated on the basis of the crystal unit cell proposed by Holmes. ${ }^{16}$ As reported by Heuvel et al., ${ }^{17}$ however, the peak positions must be selected arbitrarily without large deviation from the theoretical values in order to carry out peak separation smoothly. In this experiment, each peak position was in the reasonable ranges pointed out by them. The values of $\theta_{1}$ and $\theta_{2}$ denoting the tails of a peak at lower and higher diffraction angles were selected arbitrary, based on the method of Heuvel et $a l .{ }^{17}$ The (200) diffraction contained the contribution of the (040) diffraction because of the difficulty in resolving the superposed peak. The contribution of the (040) plane becomes more pronounced with increasing $\theta_{j}$ and the peak position shifts to higher angle from $20.4^{\circ}$ at $\theta_{j}=90^{\circ}$ to $20.8^{\circ}$ at $\theta_{j}=0^{\circ}$.

The resultant orientaiton distribution function $2 \pi q_{j}\left(\cos \theta_{j}\right)$ of the reciprocal lattice vector of the (200) plane contains the contribution from that of the (040) diffraction. The superposed function containing the contribution of both the crystal diffractions showed two peaks at $\theta_{j}=90^{\circ}$ corresponding to the (200) diffraction and $\theta_{j}=0^{\circ}$ corresponding to the (040) plane. Thus, the derivation of the (200) and (040) diffractions was performed assuming that each distribution function has a smooth distribution function along $\theta_{j}$. This treatment was carried out by a method of trial and error as shown in Figure 2 and the accuracy of the derivation shall be discussed later in terms of the second order orientation factor using 
Wilchinsky's equation. ${ }^{18}$ The derivation of the (200) and (002) plane was impossible and the contribution from the (202) plane was neglected. The second order orientation factor $F_{20}^{j}$ of the reciprocal lattice vector of the $j$-th plane may be given by

$F_{20}^{j}=\int_{0}^{\pi}(1 / 2)\left(3 \cos ^{2} \theta_{j}-1\right) 2 \pi q_{j}\left(\cos \theta_{j}\right) \sin \theta_{j} \mathrm{~d} \theta_{j}$

(2)

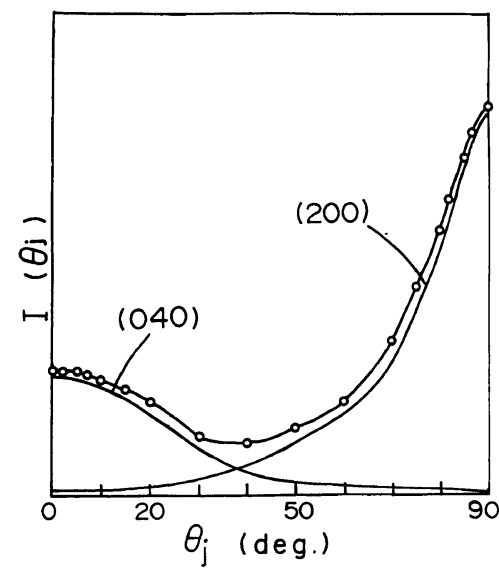

Figure 2. The contributions of the (200) and (040) planes, to the total intensity distribution function as a function of $\theta_{j}$ of the gel film with $\lambda=4$.
Estimation of the orientation of amorphous chain segments was made from the birefringence determined by subtraction of the amorphous contribution from the total birefringence, assuming simple additivity. That is,

$$
\Delta_{\text {total }}=X_{c} \Delta_{c}+\left(1-X_{c}\right) \Delta_{a}
$$

where $\Delta_{\text {total }}$ is the total birefringence of the bulk specimen, $\Delta_{c}$ is the crystalline birefringence, $\Delta_{a}$ is the amorphous birefringence, and $X_{c}$ is the valume crystallinity. In eq 3 , the contribution of the form birefringence is neglected. The crystalline birefringence $\Delta_{c}$ may be evaluated from the following relations:

$$
\Delta_{c}=n_{a} F_{20}^{a}+n_{[002]} F_{20}^{[002]}+n_{b} F_{20}^{b}
$$

$F_{20}^{a}$ and $F_{20}^{b}$ denote the second order orientation factors of the $a$ - and $b$-axes and $F_{20}^{[002]}$ the orientation factor of the reciprocal lattice vector of the (002) plane. The principal refractive indices $n_{a}, n_{[002]}$, and $n_{b}$ can be estimated in the usual way assuming the atomic arrangements within the crystal unit reported by Holmes $^{13}$ and the values of the bond polarizabilities according to Bunn and Daubeny ${ }^{19}$ and neglecting an additional bond polarizability of the hydrogen bonding. Thus, the follow-

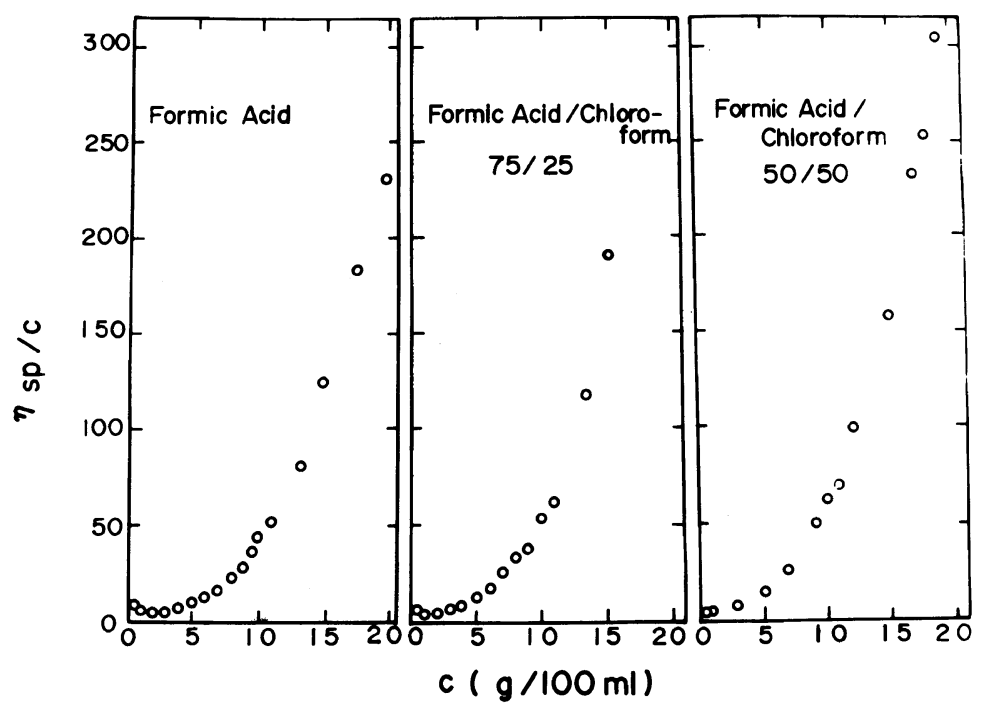

Figure 3. Relationship between reduced viscosity $\eta_{\mathrm{sp}} / c$ and concentration $c$ for three kinds of solutions of nylon 6. 


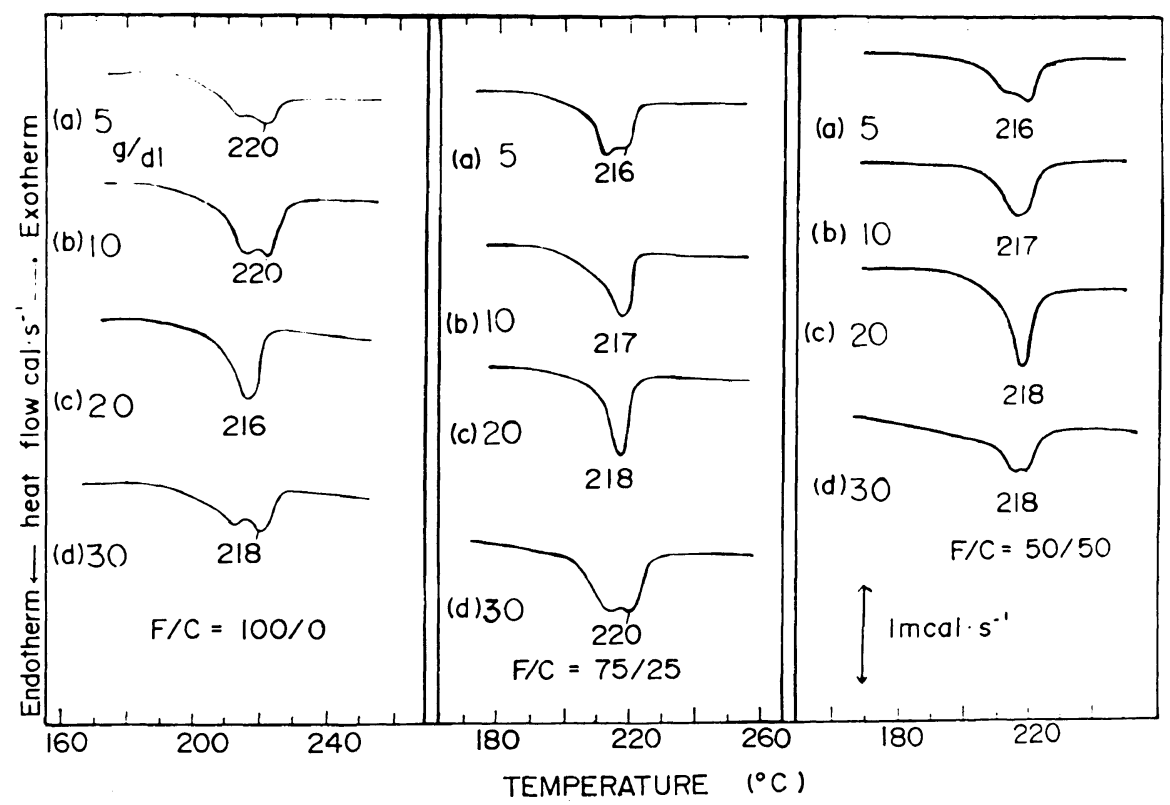

Figure 4. DSC curves of gel films prepared from solutions, in which the $\mathrm{F} / \mathrm{C}$ composition of solvent and the concentration vary as parameters.

$$
F / C=75 / 25
$$
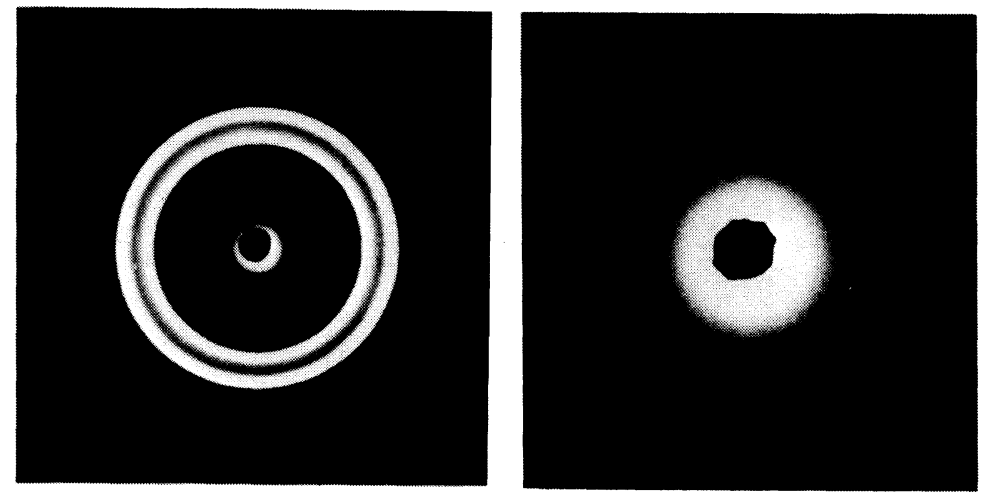

Figure 5. WAXD and SAXS patterns from an undrawn gel film with the $75 / 25$ composition (end view).

ing principal polarizabilities were obtained.

$P_{a}$ (the $a$-axis direction) $=127.9 \times 10^{-25} \mathrm{~cm}^{3}$

$P_{\text {[002] }}$ (the direction perpendicular to the (002) plane) $=110.6 \times 10^{-25} \mathrm{~cm}^{3}$

$P_{b}$ (the $b$-axis direction) $=136.0 \times 10^{-25} \mathrm{~cm}^{3}$
Then, the principal refractive indices were estimated by the Lorenz-Lorenz relation, which is given as $n_{a}=1.554, n_{[002]}=1.519$, and $n_{b}=1.669$. In this calculation, the density of nylon 6 crystal is taken as $1.23 .{ }^{16}$

The amorphous birefringence $\Delta_{a}$ may be given as 


\section{Gel Film $F / C=75 / 25$}

$\lambda=1$
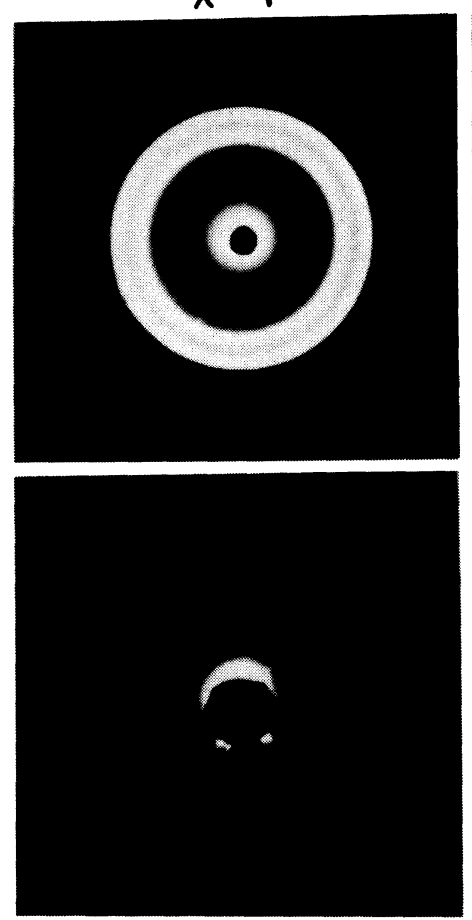

$\lambda=2$
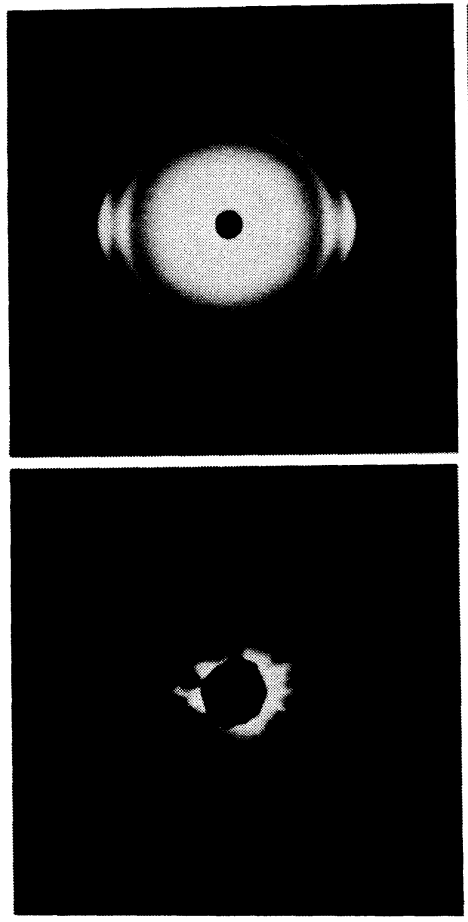

$\lambda=4$
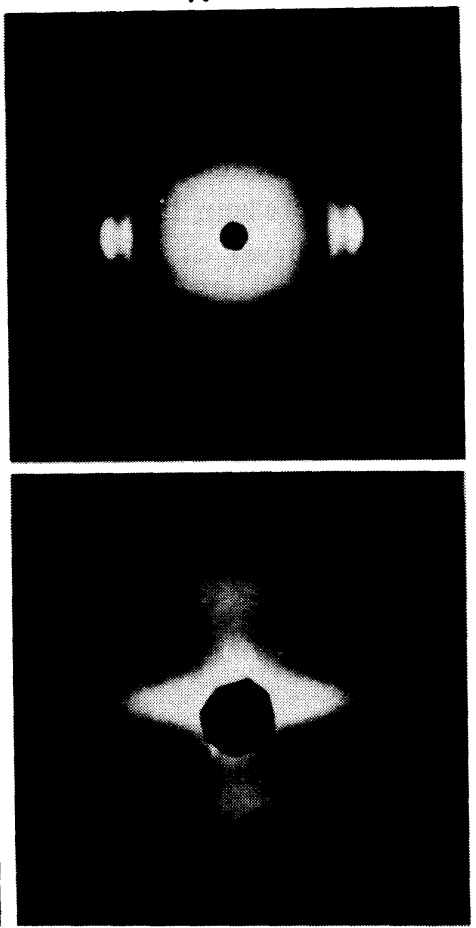

Figure 6. WAXD and SAXS patterns from undrawn and drawn gel films (through view).

$$
\Delta_{a}=F_{200}^{\mathrm{am}} \Delta_{a}^{\circ}
$$

where $F_{200}^{\mathrm{am}}$ is the second order orientation factor of the amorphous chain segments and $\Delta_{a}^{\circ}$ is given by

$$
\Delta_{a}^{\circ}=n_{\|}^{\mathrm{am}}-n_{\perp}^{\mathrm{am}}
$$

where $n_{\|}^{\text {am }}$ and $n_{\perp}^{\text {am }}$ are the refractive indices parallel and perpendicular to the chain axis. The indices, assuming rotational ellipsoidal anisotropy around the chain axis, were estimated from the polarizabilities, the difference in density from that of the crystaline phase being taken into account. This procedure gave values of $n_{\|}^{\text {am }}$ and $n_{\perp}^{\text {am }}$ as 1.582 and 1.470 , respectively, taking the density of the amorphous phase as $1.09 .^{20}$

\section{RESULTS AND DISCUSSION}

Figure 3 shows profiles of $\eta_{\mathrm{sp}} / c\left(\eta_{\mathrm{sp}}\right.$ : the specific viscosity and $c$ : concentration of solution) against $c$ to discuss the dependence of the viscosity on the composition of solvent. For three kinds of solvents, the values of $\eta_{\mathrm{sp}} / c$ show gradual increase in the concentration range up to $5 \mathrm{~g} / 100 \mathrm{ml}$ but beyond this concentration, considerable increase. As shown in Figure 3, the introduction of chloroform causes a significant increase in viscosity, especially at higher concentration and this tendency is pronounced for the solution with the $75 / 25$ composition. However, it turned out that in spite of the different viscosities with the $\mathrm{F} / \mathrm{C}$ compositions, the solutions with concentrations less than $5 \mathrm{~g} / 100 \mathrm{ml}$ have disadvantage to produce gel films and the resultant dry gels 
are composed of fibrillar structures. In contrast, good gel films with smooth surfaces could be prepared from solutions having a concentration range from 10 to $30 \mathrm{~g} / 100 \mathrm{ml}$. The gel prepared from solutions beyond $40 \mathrm{~g} /$ $100 \mathrm{ml}$, however, could not be realized to form good films, because of high viscosity.

Figure 4 presents the change in the profile of the DSC curves of gel films prepared from solutions with different kinds of concentrations. To check the reappearance of the profile, DSC measurements at a give concentration for each $\mathrm{F} / \mathrm{C}$ composition were carried out three times. The profiles are very sensitive to the concentration but independent of the $F / C$ compositions. Namely, for all compositions, the DSC curves show a single peak at $20 \mathrm{~g} /$ $100 \mathrm{ml}$ and the peak profile becomes duller having double peaks or a shoulder as the concentration departs from $20 \mathrm{~g} / 100 \mathrm{ml}$.

Table I presents the change in crystallinity with increasing the concentration of solution for the indicated F/C compositions. The crystallinity of gel films prepared from only formic acid (100/0) decreases with increasing concentration but even at $30 \mathrm{~g} / 100 \mathrm{ml}$, the value is higher than those of another films with different $\mathrm{F} / \mathrm{C}$ compositions. The introduction of chloroform causes a significant decrease in crystallinity of the original films. The crystallinity of the gel films with the $75 / 25$ composition shows a little decrease in the range $5-10 \mathrm{~g} / 100 \mathrm{ml}$ but a further increase beyond $10 \mathrm{~g} / 100 \mathrm{ml}$ is independent of crystallinity. The crystallinity of the gel with the $50 / 50$ composition increases when the concentration is beyond $10 \mathrm{~g} / 100 \mathrm{ml}$.

In spite of the experimental results in Figures 3-4 and Table I, we could not find suitable conditions to prepare gels with high drawability. Accordingly, as a method of trial and error, resultant gel films from solutions with different $\mathrm{F} / \mathrm{C}$ compositons and different concentrations were elongated in a hot oven at $205^{\circ} \mathrm{C}$ under nitrogen. It turned out that the possibility of successfully drawing up to 4 fold
Table I. Concentration-dependence of the crystallinity. of gel films prepared from different kinds of $\mathrm{F} / \mathrm{C}$ compositions $(\%)$

\begin{tabular}{cccc}
\hline & \multicolumn{3}{c}{ Gel films } \\
\cline { 2 - 4 } $\begin{array}{c}\text { Concentration } \\
(\mathrm{g} / 100 \mathrm{ml})\end{array}$ & $100 / 0$ & $75 / 25$ & $50 / 50$ \\
\hline 5 & 55.1 & 35.6 & 34.8 \\
10 & 52.0 & 32.4 & 34.0 \\
20 & 52.8 & 29.2 & 37.2 \\
30 & 48.2 & 30.0 & 40.4 \\
\hline
\end{tabular}

$(\lambda=4)$ depends on the concentration and the $\mathrm{F} / \mathrm{C}$ compositions, sensitively. When the films were prepared from a solution with $30 \mathrm{~g} /$ $100 \mathrm{ml}$, they could easily be elongated to $\lambda=4$. Namely, the possibility becomes lower as the concentration departs from $30 \mathrm{~g} / 100 \mathrm{ml}$. Among the specimens prepared from $30 \mathrm{~g} /$ $100 \mathrm{ml}$, the drawability also depends upon $\mathrm{F} / \mathrm{C}$ composition sensitively. The film with $75 / 25$ composition could be readily elongated up to $\lambda=4$ in comparison with the films with the $100 / 0$ and 50/50 compositions. This indicates that the high crystallinity of original film and the oriented crystallization under elongation hamper the drawability and the elongation can be mainly realized by the orientation of amorphous chain segments. This supports the preferential orientation of the $b$-axis is attributed to the rotation of crystallites but not the crystal transformation from a folded to a fibrous structure.

Table II presents the mechanical properties of the gel and melt films in an undeformed state and their maximum draw ratios. The Young's modulus and tensile strength at $\eta=1$ and 4 depend on the $\mathrm{F} / \mathrm{C}$ composition but all the values of the gel films are lower than those of.melt films. The possibility of successful drawability up to $\lambda=4$ is best for films prepared from a solution with the $75 / 25$ composition and the $\mathrm{F} / \mathrm{C}$ composition is not sensitive to the Young's modulus and tensile strength. The resultant values are not superior to the previous values. ${ }^{10,11}$ Accordingly, the follow- 
Table II. Mechanical properties of gel films with different kinds of $\mathrm{F} / \mathrm{C}$ and melt films

\begin{tabular}{lcccccc}
\hline & Draw & \multicolumn{3}{c}{ Gel films (F/C) } & Melt \\
\cline { 3 - 6 } & ratio & $100 / 0$ & $75 / 25$ & $50 / 50$ & film \\
\hline $\begin{array}{c}\text { Young's modulus } \\
(\mathrm{GPa})\end{array}$ & 1 & 1.18 & 1.17 & 1.25 & 3.60 \\
& 4 & 3.14 & 3.18 & 3.96 & - \\
& 5 & - & - & - & 4.97 \\
\hline $\begin{array}{c}\text { Tensile strength } \\
(\mathrm{MPa})\end{array}$ & 1 & 58.6 & 68.9 & 74.0 & 62.9 \\
& 4 & 170.6 & 174.3 & 197.2 & - \\
& 5 & - & - & - & 143.5 \\
\hline $\begin{array}{c}\text { Elongation at } \\
\text { break (\%) }\end{array}$ & 1 & 38.1 & 39.0 & 37.3 & 217.0 \\
& 4 & 8.29 & 14.0 & 10.5 & - \\
& 5 & - & - & - & 9.03 \\
\hline
\end{tabular}

ing morphological and mechanical properties were analyzed using the films with the $75 / 25$ composition, in order to study the cause of poor mechanical properties.

Figure 5 shows WAXD and SAXS patterns for an undrawn gel film with the $75 / 25$ composition, when the incident beam was directed parallel to the film surface (end view). The WAXD pattern shows diffraction rings and the SAXS pattern shows no scattering. The WAXD and SAXS patterns together indicate that the gel film is composed of crystallites which are oriented randomly and the crystallites do not form ordered crystal lamellar assemblies so as to give scattering rings. This mode is quite different from the structures of gel films of polyethylene $e^{6,21-23}$ and polypropylene $^{8}$ whose constituent lamellar crystals as an assembly are oriented parallel to the film surface.

Figure 6 shows WAXD and SAXS patterns for undrawn and drawn gel films, when the incident beam is directed perpendicular to the film surface (through view). The WAXD patterns indicate the preferential orientation of the $b$-axis with respect to the stretching direction. The SAXS pattern from the undrawn film shows no scattering and the patterns from the drawn films with $\lambda=2$ and 4 show meridional scattering having à scattering maximum, cor- responding to a long period of about 106 and $125 \AA$, respectively. The SAXS patterns with draw ratio indicates that the crystallites having random orientation form lamellar crystal assemblies owing to oriented crystallization under elongation process and the crystal lamellae are highly oriented with large flat faces with respect to the direction normal to the stretching direction. The WAXD and SAXS patterns together indicate that within crystal lamellae, the $b$-axes are oriented perpendicular to the large flat faces.

Figure 7 shows WAXD and SAXS patterns for undrawn and drawn melt films. The WAXD and SAXS patterns show an orientational mode similar to that for the gel fims shows in Figure 4. This indicates that the orientation of crystallites for the gel film is almost similar to that of the melt film. The long period of the melt film increases with draw ratio; $94 \AA$ at $\lambda=2,108 \AA$ at $\lambda=4$, and $115 \AA$ at $\lambda=5$. The WAXD patterns in Figures 6 and 7 show the weak diffraction spots of the (040) plane of the $\alpha$-form crystals and of the (020) plane of the $\gamma$-form crystals, respectively, in the meridional direction. As discussed before, the contribution of the (040) plane must be subtracted from the total intensity distribution to estimate the orientation behavior of the (200) plane. As for the (020) plane of the $\gamma$ form crystals, the diffraction from the gel film is extremely weak and this phenomenon is quite different from the previous report that the gel film prepared from formic acid is composed of the $\gamma$-form crystals. ${ }^{24}$

In order to estimate the contents of the $\alpha$ and $\gamma$-form crystals quantitatively, the intensity distribution was measured by a step scanning method. The step interval was fixed to be $0.2^{\circ}$ with a time interval of $4000 \mathrm{~s}$ in the range $13-32^{\circ}$ (twice the Bragg angle). In this measurement, a sample was rotated around the film normal direction in order to average the effect of crystal orientation. Table III presents the contents of the $\gamma$ - and $\alpha$-form crystals of the gel and melt films as a function of draw ratio. 
Melt pressed film

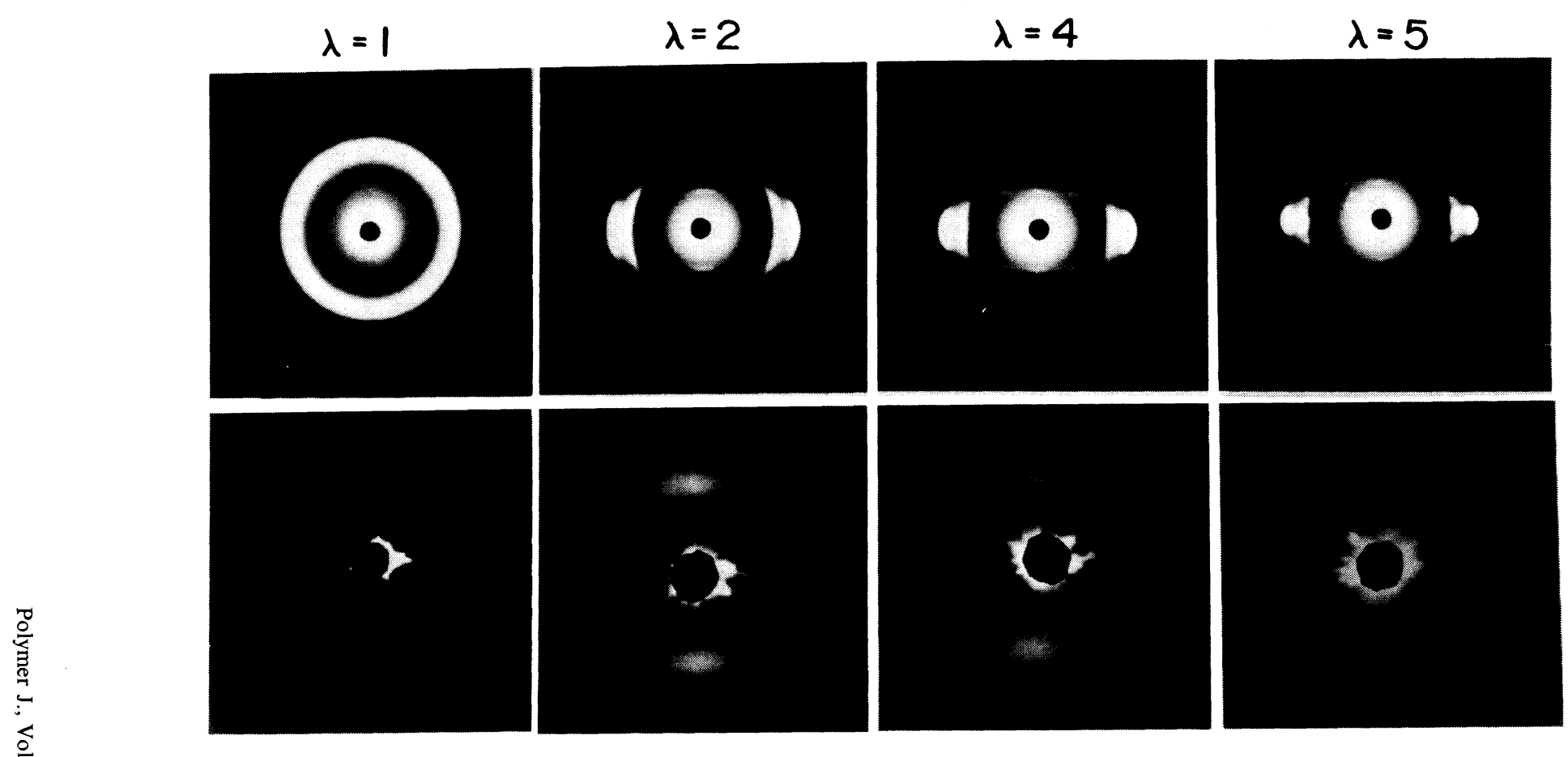

Figure 7. WAXD and SAXS patterns from undrawn and drawn melt films (through view). 
Table III. Contents of the $\alpha$ - and $\gamma$-form crystals within gel and melt films with various draw ratios $(\%)$

\begin{tabular}{lccc}
\hline Specimen & Draw ratio $(\lambda)$ & $\alpha$-Form & $\gamma$-Form \\
\hline \multirow{2}{*}{ Gel film (75/25) } & $1^{\mathrm{a}}$ & 93.1 & 6.9 \\
& 2 & 95.7 & 4.3 \\
& 4 & 96.8 & 3.2 \\
\hline \multirow{2}{*}{ Melt film } & $1^{\mathrm{a}}$ & 0 & 100 \\
& & 90.0 & 10.0 \\
& 2 & 94.0 & 6.0 \\
& 4 & 98.3 & 1.7 \\
& 5 & 99.5 & 0.47 \\
\hline
\end{tabular}

a Before annealing.

This indicates that the original melt film is composed of the $\gamma$-form crystals but the annealing for $30 \mathrm{~min}$ at $205^{\circ} \mathrm{C}$ causes $90 \%$ transformation from the $\gamma$ - to the $\alpha$-form crystals. ${ }^{15}$ The transformation is more pronounced as the draw ratio increases and at $\lambda=5$ most of crystals take on the $\alpha$-form. In contrast, the $\alpha$ form crystals of an original gel film occupy $93.1 \%$ and this content is hardly affected by annealing. The content of the $\alpha$-form crystals increases with increasing draw ratio, as in the case of the melt films.

Figure 8 shows the change in the profile of DSC curves of gel and melt films with increasing draw ratio. Prior to the DSC measurements, all gel and melt films were annealed to study the thermal behavior of the $\alpha$-form crystals. For the gel films, the melting endotherm peak position and shape depend on draw ratio. The peak position shifts to higher temperature and the endotherm of curves is more intense with increasing draw ratio. In contrast, for the melt films, the shape of endotherm becomes more definite but the peak position shifts to lower temperature with increasing draw ratio. The latter tendency is quite different from the thermal behavior of the gel films.

In order to understand this difference, the crystallinities of the gel and melt films were obtained as a function of draw ratio. Table IV

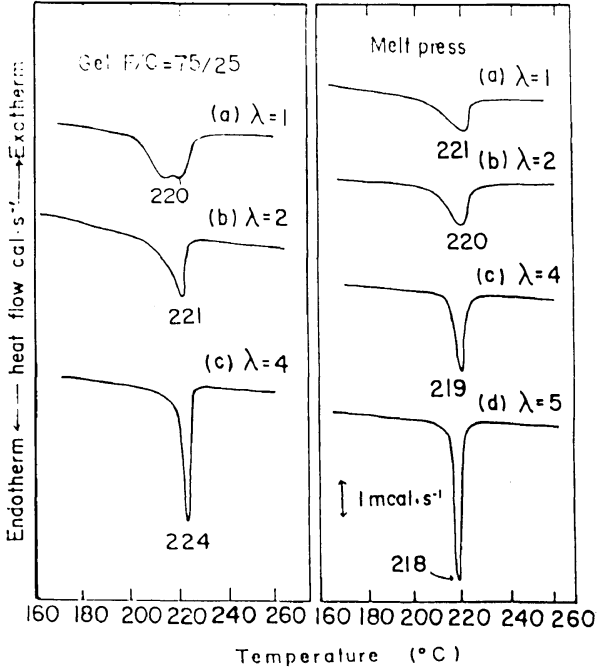

Figure 8. DSC curves of gel and melt films with various draw ratios.

Table IV. Crystallinity of gel and melt films with various draw ratios

\begin{tabular}{ccc}
\hline \multirow{2}{*}{ Draw ratio $(\lambda)$} & \multicolumn{2}{c}{ Crystallinity/\% } \\
\cline { 2 - 3 } & Gel film $(75 / 25)$ & Melt film \\
\hline 1 & 30.0 & 25.1 \\
2 & 41.4 & 38.8 \\
4 & 47.4 & 41.9 \\
5 & - & 46.6 \\
\hline
\end{tabular}

shows that crystallinity increases with the draw ratio. The increase in crystallinity causes a significant change in DSC data in Figure 8. As discussed before, the peak profile is more intense with increasing crystallinities of gel and melt films. The problem that the peak of the melt film shifts to lower temperature with increasing draw ratio, however, cannot be resolved in terms of the change in crystallinity. Accordingly, the effect of an unknown contribution must be taken into account in further studies.

SALS studies were carried out on the undrawn and drawn melt films under $H_{\mathrm{v}}$ polarization condition. Figure 9 shows the patterns. The scattering from the gel films could not be obtained because of the difficulty in observing 


\section{melt pressed film}

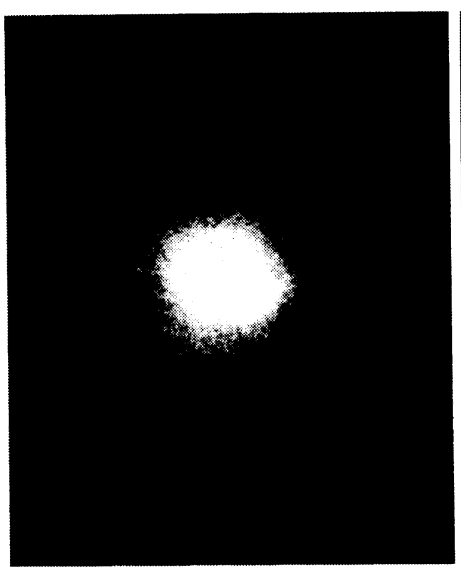

$$
\lambda=1
$$

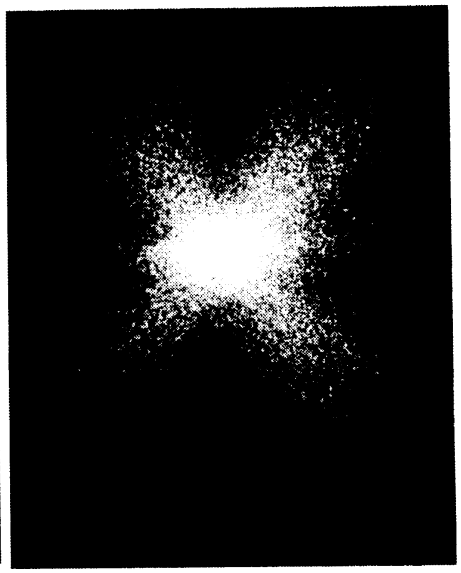

$\lambda=2$
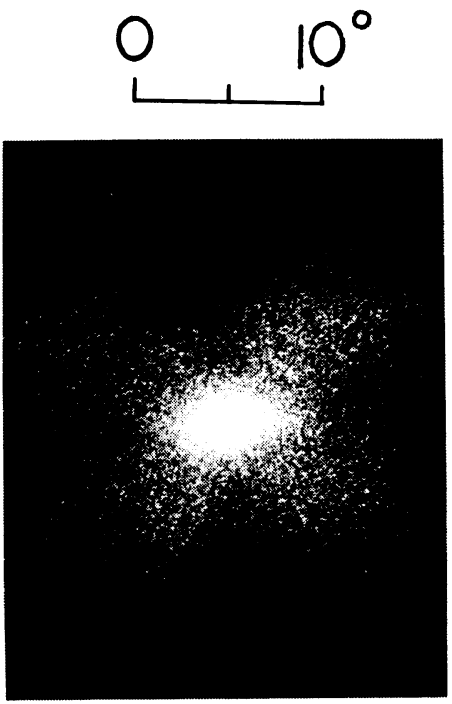

$\lambda=5$

Figure 9. SALS patterns from undrawn and drawn melt films under $H_{v}$ polarization.

clear patterns for the whitening. The scattering from the undrawn film displays a circular pattern in that the intensity continuously decreases with increasing scattering angle but does not vary with the azimuthal angle. This is typical of the scattering from a system being a random array of crystallites that are small compared with the wavelength of incident beam. Elongation causes development of the X-type $H_{\mathrm{v}}$ patterns. The intensity had a maximum in the scattering center and it decreased monotonically with increasing scattering angle. These observations are indicative of scattering from rodlike textures. The pattern (b) reveals that the rods take on random orientation or are oriented at $\pm 45^{\circ}$ with respect to the stretching direction. Birefringence data at $\lambda=2$ and 4 are $36.2 \times 10^{-3}$ and $64.4 \times 10^{-3}$, respectively. This indicates that the rods at $\lambda=2$ are oriented at $\pm 45^{\circ}$ with respect to the stretching direction and the optical axes within the rod are oriented predominantly to the stretching direction. The formation of rods is probably due to the oriented crystallization. With further elonga-
Table V. The second order orientation factors of the reciprocal lattice vectors of the (200), (002), and (040) planes for gel and melt gilms with various draw ratios

\begin{tabular}{ccccc}
\hline \multirow{2}{*}{ Specimen } & \multirow{2}{*}{$\begin{array}{c}\text { Draw } \\
\text { ratio }\end{array}$} & \multicolumn{3}{c}{ The second order orientation factor } \\
\cline { 3 - 5 } & & $(200)$ & $(002)$ & $(040)$ \\
\hline Gel film & 2 & -0.216 & -0.352 & 0.527 \\
$(75 / 25)$ & 4 & -0.336 & -0.414 & 0.611 \\
\hline \multirow{2}{*}{ Melt film } & 2 & -0.262 & -0.321 & 0.528 \\
& 4 & -0.282 & -0.418 & 0.657 \\
& 5 & -0.350 & -0.413 & 0.716 \\
\hline
\end{tabular}

tion up to $i=4$, the scattering lobes in pattern (c) are extended in the horizontal direction, indicating preferential orientation of rods with respect to the stretching direction.

Table V shows the second order orientation factors of the (200), (002), and (040) planes and Table VI the second order orientation factors of the principal crystallographic axes. The factors of the $a$ - adn $c$-axes in Table VI were calculated from those of the reciprocal lattice vector of the (200) and (002) planes 
Table VI. The second order orientation factors of the three principal crystallographic axes and amorphous cahin segments, in which the orientation factor of the $b$-axis is calculated using Wilchinsky's equation on the basis of the factors concerning the (200) and (002) planes

\begin{tabular}{lcccccc}
\hline & & \multicolumn{5}{c}{ The second order orientation factor } \\
\cline { 3 - 6 } Specimen & Draw ratio & $a$-axis & $b$-axis & $c$-axis & $\begin{array}{c}\text { Amorphous chain } \\
\text { segment }\end{array}$ & $\begin{array}{c}b \text {-axis (calculated by } \\
\text { Wilchinsky's equation) }\end{array}$ \\
\hline \multirow{2}{*}{ Gel film (75/25) } & 2 & -0.192 & 0.527 & -0.329 & - & 0.517 \\
& 4 & -0.272 & 0.667 & -0.393 & - & 0.686 \\
\hline \multirow{2}{*}{ Melt film } & 2 & -0.252 & 0.528 & -0.311 & 0.573 & 0.573 \\
& 4 & -0.259 & 0.657 & -0.395 & 0.743 & 0.677 \\
& 5 & -0.340 & 0.716 & -0.402 & 0.856 & 0.752 \\
\hline
\end{tabular}

using Wilchinsky's equation. ${ }^{18}$ The orientation factor of the $b$-axis corresponds to that of the reciprocal lattice vector of the (040) plane. As discussed before, however, the peak separation concerning the (200) and (040) planes is very difficult because of the adjacent values of diffraction angles. In order to check the accuracy of the peak separation, the orientation factor of the $b$-axis was calculated from those of the reciprocal lattice vectors of the (200) and (002) planes using Wilchinsky's equation ${ }^{18}$ given on the right side of Table VI. The factors estimated directly from the reciprocal lattice vector of the (040) plane are in good agreement with those on the right hand and this supports the accuracy of the peak separation.

As listed in Table VI, the orientation factor indicates that the $b$-axes orient predominantly with respect to the stretching direction, while the $c$-axes orient perpendicular, as draw ratio increases. The difference of the factors between the $a$ - and $c$-axes indicates that the $a$ - and $c$ axes do not assure random orientation around the $b$-axis. That is, the orientational degree of the $c$-axis is higher than that of the $a$-axis. This means that the crystal orientations within drawn gel and melt films are different from the perfect uniaxial orientation mode and the preferential orientation of the $b$-axis with respect to the stretching direction is associated with the rotation of crystallites leading to taut tie molecules. Actually, the orientational degree of amoprhous chain segments for the melt films is higher than that of the $b$-axes. This assumption for the orientational mode supports the high orientational degree of the $b$ axis at initial draw ratio in Table VI. If crystal transformation from a folded to a fibrous structure occurs during elongation process, it would be expected that molecules almost retain a random orientation at lower draw ratios such as $i=2$ and 4 and an extremely high orientation of the $b$-axis can be realized with further elongation up to high draw ratio. Such an ideal orientation of the crystal fiber axis to assure high drawability has been observed for polyethylene and polypropylene gel films. ${ }^{3-8}$

\section{CONCLUSION}

Nylon 6 films were prepared by gelation/ crystallization from solutions and by modling from melt state. The resultant gel and melt films were elongated in an hot oven at $205^{\circ} \mathrm{C}$ under nitrogen. In preparing gel films, the $\mathrm{F} / \mathrm{C}$ compositions of co-solvent mixtures chosen were $100 / 0,75 / 25$, and $50 / 50$. The mechanical and morphological properties of the resultant gel films are almost independent of the $\mathrm{F} / \mathrm{C}$ compositions. The Young's modulus and tensile strength of all the films are less than 5 and $0.15 \mathrm{GPa}$, respectively. These values were not superior to those reported by Gogolewski et $a l{ }^{11}$ for gel deformation method and by 
Kunugi et al. for zone-annealing of commerical nylon 6 fibers. ${ }^{25-27}$

Such poor mechanical properties in the current work are attributed to the fact that the preferential orintation of the $b$-axis is due to the rotation of crystallites but not to the crystal transformation from a folded to a fibrous structure. The crystal transformation during elongation is an important factor to assure high drawability as in the case of polyethylene and polypropylene gel films.

\section{REFERENCES AND NOTES}

1. J. Smook, J. C. Torf, P. F. van Hutter, and A. J. Pennings, Polym. Bull., 2, 293 (1980).

2. P. J. Barham and A. Keller, J. Material Sci., 15, 2229 (1980).

3. T. Kanamoto, A. Tsuruta, K. Tanaka, M. Takeda, and R. S. Porter, Polym. J., 15, 327 (1983).

4. K. Furuhata, T. Yokokawa, and K. Miyasaka, J. Polym. Sci., Polym. Phys. Ed., 22, 133 (1984).

5. P. Smith, P. J. Lemstra, and H. C. Booij, J. Polym. Sci., Polym. Phys. Ed., 19, 877 (1981).

6. P. Smith, P. J. Lemstra, J. P. L. Pippers, and A. M. Kiel, Colloid Polym. Sci., 258, 1070 (1981).

7. M. Matsuo and C. Sawatari, Macromolecules, 19, 2036 (1986).

8. M. Matsuo, C. Sawatari, and T. Nakano, Polym. J., 18, 759 (1986).

9. C. Sawatari and M. Matsuo, Macromolecules, 19, 2653 (1986).

10. H. H. Chauh and R. S. Porter, Polymer, 27, 1022 (1986).

11. S. Gogolewski and A. J. Pennings, Polymer, 26, 1394 (1985).

12. K. Kaji and I. Sakurada, Macromol. Chem., 179, 209 (1978).

13. According to ref 11 , the relationship to obtain average molecular weight of nylon 6 by Gogolewski and Pennings was in private communication with Dr. J. A. Juijn of Enka Research, Arnhem, Holland.

14. H. Arimoto, J. Polym. Sci., A-2, 2283 (1964).
15. J. P. Parker and P. H. Lindenmeyer, J. Appl. Polym. $S c i ., 21,821$ (1977). This paper reported that in the $\beta$ form the fiber repeat distance is in excess of $17 \AA$ and the crystallographic $\beta$ angle is $60^{\circ}$ or greater, while in the $\gamma$-form the distance is about $16.8 \AA$ and the angle $\beta$ is $60^{\circ}$ or less. In the former type, the hydrogen bonds are between antiparallel chain which are almost extended and in the latter type, the amide groups are twisted out of the plane of the methylene group. According to this report, the $\beta$-form is unstable and changes to the $\alpha$-form very easily. However, Parker and Lindenmeyer did not report whether the $\gamma$-form crystals in undeformed and unannealed state retain even under elongation and annealing. In our experiment, the peaks to distinguish the $\beta$ - and $\gamma$ form crystals cannot be detected by the $X$-ray diffraction intensity in the range $74-82^{\circ}$ (twice the Bragg angles) because of a very smaller amount of the $\gamma$ form (or the $\beta$-form) crystal content in comparison with the $\alpha$-form crystal content. Furthermore the Xray diffraction profile in unannealed melt film in our experiment is of same type in Figure 3(b) in this reference. For simplicity we shall neglect the existence of the $\beta$-form crystals.

16. D. R. Holmes, C. W. Bunn, and D. J. Smith, J. Polym. Sci., 17, 159 (1955).

17. H. M. Henvel and R. H. Akzo, J. Polym. Sci., Polym. Phys. Ed., 19, 121 (1981).

18. Z. W. Wilchinsky, J. Appl. Phys., 31, 1969 (1960).

19. C. W. Bunn and R. P. Daubeny, Trans. Faraday Soc., 50, 1173 (1954).

20. E. Balcerzyk, W. Kozlowski, E. Wesolowska, and W. Lewaszkiewicz, J. Appl. Polym. Sci., 26, 2573 (1981).

21. M. Matsuo and R. St. J. Manley, Macromolecules, 15, 985 (1982).

22. M. Matsuo, C. Sawatari, M. Iida, and M. Yoneda, Polym. J., 17, 1197 (1985).

23. T. Ohta, O. Yoshizaki, and E. Nagai, Kobunshi Kagaku, 20, 225 (1963).

24. W. P. Leung, K. H. Ho, and C. L. Choy, J. Polym. Sci., Polym. Phys. Ed., 22, 1173 (1984).

25. T. Kunugi, I. Akiyama, and M. Hashimoto, Polymer, 23, 1193 (1982).

26. T. Kunugi, I. Akiyama, and M. Hashimoto, Polymer, 23, 1199 (1982).

27. T. Kunugi, T. Ikuta, and M. Hashimoto, Polymer, 23, 1983 (1982). 